\section{NOTES ON A CASE OF}

\section{RESECTION OF TIBIA WITH GRAFTING. WITH PLATING OF FRACTURED GRAFT.}

BY L. E. BARRINGTON-WARD, CH.M., F.R.C.S. EDIN., F.R.C.S. ENG.,

SURGEON (IN CHARGE OF OUT-PATIENTS) TO THE HOSPITAL FOR SICK CHILDREN, GREAT ORMOND-STREET, AND GREAT NORTHETN CENTRAL HOSPITAL.

IN the operative treatment of chronic osteomyelitis of a long bone two alternative lines of treatment are available. Chronic osteomyelitis is not here taken to mean the chronic condition resulting from the unsuccessful treatment of acute osteomyelitis, but quiet bone inflammation of staphylococcal, pneumococcal, or tuberculous origin. (These are, as a rule, indistinguishable clinically and radiographically, and in most cases can only be differentiated after bacteriological examination.) The oldest method is the simplest, the most generally used, and perhaps the least efficacious. An incision is made over the affected bone, the medullary cavity is opened up, diseased bone is removed by gouge and forceps until healthy limits are reached, the resulting cavity is disinfected thoroughly, the soft parts are brought together, and the wound is closed.

The second method has been most strongly advocated and developed by Stiles of Edinburgh. This is more radical, and the diseased shaft is removed entire. By appropriate splinting the limb is kept in good position, and in the course of time the diaphysis is reproduced in healthy bone.

\section{The Method Used.}

A third method is a modification and, I believe, an improvement on the simple resection. It consists in excision of the affected shaft through healthy limits and inserting a bone-graft-generally obtained from the tibia-into the gap so made. This transplant is cut to the exact length required, and is fashioned and fixed as an efficient internal splint, rendering unnecessary the subsequent use of cumbrous apparatus, and helping to some extent in the regeneration of bone. It is also possible that the fresh healthy bone cells of the graft may assist in dealing with any of the original infection - for example, of the pexiosteum - which has inadvertently or unavoidably been left behind.

Various grafts have been suggested-the tibia from a recently amputated leg, the fibula of the same patient. Such complications are unnecessary. A strip from the patient's healthy tibia fulfils all requirements, is easily cut with simple instruments, and has no ill-effects on the donor. It is not necessary to take periosteum with the graft. The true periosteum is a limiting membrane, and, provided the periosteal tube is left to receive the graft, the perfect shape of the bone will be reproduced. I have employed this method with complete suceess in treating osteomyelitis of the humerus, radius, ulna;" and tibia. The following case illustrates the method in the case of the tibia, but it is recorded rather to show the behaviour of the graft under.trying circumstances.

The patient, a girl aged 6, came under my care at the Hospital for Sick Children, Great Ormond-street, in March, 1914: She had a swelling of the middle third of the tibia, which had been noticed for a year. There was no history of injury: The Wassermann reaotion was negative.: $X$ ray: showed a dense osteitis of the shaft of the right tibia. ; At operation on April 2nd the selerosed bone was opened up and an abscess cavity was found in the centre. The pus was. sterile, and no tubercle bacilli could be found. The diseased bone was removed as far as possible, the cavity disinfected, and the wound closed without drainage. The wound healed satisfactorily, and progress was uninterrupted until July, 1914. A recrudescence of activity was then noticed, the bone becoming more swollen and tender. She was again admitted, and an operation: 'was performed at a denionstration before the American 'Congress of Surgeons'

The onliginal scar: was excised and about 6 .inches: of the tibial shaft, including all the diseased bone, was resected subperiosteally. The medullafy cavity at each end was reamed out and careful measurements were taken...A gralt was then cut of the required length and size from the normal tibia and shaped to fit the gap exactly. Each end of the graft was rammed firmly into the reamed out medullary cavity, a shoulder being "fashioned at either end to ensure correct length. The wounds were closed and the leg put in plaster. $\mathrm{X}$ rays from time to time showed the good progress of the transplant. It would seem that growth was taking place chiefly from the cut ends of bone along the scaffold graft. By February, 1916, the patient was walking about without a splint. In March, 1916, she fell over a railing and snapped the graft across. She was readmitted to hospital and the fracture was accurately set by open operation and plated by Lane's method. Subsequent history was uneventful. She was allowed to walk freely four months later. When last examined (September, 1919) she had a normal right leg, equal in length, size, and strength to the left, and perfect function.

\section{Remarks.}

This case illustrates some interesting points in the question of bone regeneration. The opportunity was taken when the fractured graft was plated to make a careful examination of the twenty-month-old transplant. The graft was tapered from either end and had broken at its thinnest part-the middle. It would seem that most of the growth had taken place at the ends, and that bone had grown down and up along the graft. There was no evidence yet of a medullary cavity. The texture of the bone was firm and the screws gripped well. In the growth of the transplant since the plating the plate has remained on the surface, showing that the increased thickness was not due to deposition of bone by the periosteum.

The fracture, as is usual with plated fractures, healed without external callus. The last $X$ ray, five and a half years after the original operation, showed perfect reproduction of the shaft with restoration of the medullary cavity and no trace of graft or fracture.

\section{A CLINICAL STUDY OF}

\section{INFECTIONS OF THE HAND.}

\section{BY H. W. L. MOLESWORTH, F.R.C.S. ENG.,}

\section{SENIOR HOUSE SURGEON, POPLAR HOSPITAL FOR ACCIDENTS.}

LITTLE has been written in English publications on this important subject, which has, however, been reviewed very completely in America by Kanavel-an author too little known in this country. This investigation has been made in order to show how the graver hand infections commence and to ascertain whether these severe lesions are preventable by early appropriate treatment. As certain facts have been observed which, though not bearing directly upon this matter, may be of interest, the paper has developed into a brief account of infections of the hand as seen in those who earn their living with their hands. Every available case, trivial or otherwise, was seen, notes were kept, and treatment was either supervised or carried out by the writer; the severer cases were admitted to beds which were under his direct observation. I should like to compare the results in this series of cases with those obtained among the nursing staff of a large institution, where cases report sick early, and consequently get early and thorough treatment.

\section{Anatomy.}

In order that the descriptions of fascial space infections may be understood the briefest possible account of the anatomy is given, as this is not well described in text-books of anatomy. Those who wish will find an extremely full account in Kanavel's "Infections of the Hand."

There are six well-defined spaces which are liable to become infected.

1. Dorsal subcutaneous. 2. Dorsal subaponeurotic. These two spaces on the dorsum of the hand and fingers are sufficiently well described by their names.

3. The middle palmai space. This lies deep to the flexor tendons and superficial to the metacarpals and interossei ; it is limited on itś radial side by a layer of fascia at the level of the third metacarpal bone. It is 